\title{
Dose-escalation phase I study in metastatic breast cancer patients with combination of paclitaxel and tegafur'uracil
}

\author{
AKIHIKO OSAKI ${ }^{1,7}$, SHOSHU MITSUYAMA ${ }^{2}$, JUN-ICHI KUREBAYASHI ${ }^{3}$, HIROSHI SONOO ${ }^{3}$, \\ REIKI NISHIMURA ${ }^{4}$, TOSHIHIRO KOGA ${ }^{5}$, SHIGERU MURAKAMI ${ }^{1}$ and SHINJI OHNO ${ }^{6}$ \\ ${ }^{1}$ Department of Surgical Oncology, Research Institute for Radiation Biology and Medicine, \\ Hiroshima University, Hiroshima-shi, Hiroshima 734-8551; ${ }^{2}$ Kitakyushu Municipal Medical Center, \\ Kitakyushu-shi, Fukuoka 802-0077; ${ }^{3}$ Department of Breast and Thyroid Surgery, Kawasaki Medical School, \\ Kurashiki-shi, Okayama 701-019; ${ }^{4}$ Department of Surgery, Kumamoto Municipal Hospital, Kumamoto-shi, \\ Kumamoto 862-0909; ${ }^{5}$ Hirose Hospital, Fukuoka-shi, Fukuoka 810-0004; ${ }^{6}$ Department of Breast Oncology, \\ National Kyushu Cancer Center Hospital, Fukuoka-shi, Fukuoka 811-1395, Japan
}

Received April 24, 2009; Accepted August 7, 2009

DOI: 10.3892/ol_00000008

\begin{abstract}
The study present the results of the dose-setting study of concomitant weekly administration of paclitaxel and tegafur-uracil (UFT) for metastatic breast cancer. Eligible patients who entered the study underwent two or more courses of weekly paclitaxel + UFT therapy as the protocol therapy. The initial dose (level 1) was paclitaxel, $80 \mathrm{mg} / \mathrm{m}^{2}$ and UFT, $400 \mathrm{mg} /$ day. At level 2, paclitaxel remained the same, but UFT was increased to $600 \mathrm{mg} /$ day. At level 3, only paclitaxel was increased to $90 \mathrm{mg} / \mathrm{m}^{2}$. Twelve patients were enrolled in this study between September 2000 and September 2002. Three patients were assigned to level 1 . Grade 3 liver dysfunction (increased aspartate aminotransferase and alanine aminotransferase) was noted in one patient and grade 4 neutropenia was noted in one patient, showing that dose-limiting toxicity was detected in 2/3 patients. In accordance with the protocol, UFT was fixed at $400 \mathrm{mg} /$ day and paclitaxel was decreased to $60 \mathrm{mg} / \mathrm{m}^{2}$ at level -1 , and then increased to $70 \mathrm{mg} / \mathrm{m}^{2}$ at level 0 . The overall effective rate after completion of two courses was $33 \%$ (3/9) including one case of complete response and two cases of partial responses. The remaining patients presented with stable diseases and no patient had progressive disease. In this study, weekly paclitaxel with concomitant UFT was administered. The recommended doses of paclitaxel and UFT were determined to be $70 \mathrm{mg} / \mathrm{m}^{2}$ and $400 \mathrm{mg} /$ day, respectively. As the toxicity profile shows, the highest toxicity level of this
\end{abstract}

Correspondence to: Dr Akihiko Osaki, Present address: ${ }^{7}$ Department of Breast Oncology, Saitama Medical University, 1397-1 Yamane, Hidaka-shi, Saitama 350-1298, Japan

E-mail: aosaki@saitama-med.ac.jp

Key words: uracil and tegafur, paclitaxel, phase I, metastatic breast cancer regimen was neutropenia and liver dysfunction, and doselimiting toxicity was neutropenia.

\section{Introduction}

An anthracycline-containing regimen represents the first-line palliative chemotherapy (1-3). However, it is necessary to develop non-anthracycline combination regimens to provide salvage therapy in metastatic breast cancer patients who have relapsed during or after anthracycline-containing combinations. Although new salvage chemotherapy using (or in combination with) novel anticancer drugs has been studied (4-6), survival benefits and higher response rates are often countered by increased toxicity and complexity of regimen. An effective combination chemotherapy regimen that is both simple and has lesser toxicity would be valuable.

Paclitaxel is highly effective for both breast cancer without previous treatment (7) and breast cancer previously treated with anthracycline (8). Findings in a Japanese late phase II study showed the effective rate in metastatic breast cancer patients to be $33.7 \%$ (21/62) (9). Clinical evaluation of weekly regimens was frequently performed. Higher effects with mild adverse events compared to those of the approved dosage/application method, comprising an every-three-week regimen, have also been reported (10). These regimens can be administered on an outpatient basis, which is an advantage.

5-Fluorouracil is used in combination with anthracycline anticancer drugs and cyclophosphamide for the treatment of breast cancer and is administered by bolus injection in many cases. However, continuous intravenous infusion is the best administration method because the effect of 5-fluorouracil is time-dependent and increases with the duration of exposure of tumor cells (11). Several reports have shown that continuous intravenous infusion was effective for colon carcinoma.

Thus, we paid attention to tegafur-uracil (UFT) because its oral administration obtains area under the curve comparable to that obtained by continuous intravenous infusion of 5-fluorouracil. UFT is an anticancer drug developed in Japan and consists 
of the masked compound of 5-fluorouracil (tegafur) and uracil. UFT inhibits the rate-limiting decomposition enzymes of 5-fluorouracil that is dihydropyrimidine dehydrogenase (DPD). UFT has tegafur and uracil at a molar ratio of 1:4. The effects of UFT alone on local progressive and metastatic breast cancer were reported to be 32 (12) and 39\% (13), respectively.

This study presents the results of the dose-setting study of concomitant weekly administration of paclitaxel and UFT for metastatic breast cancer.

\section{Patients and methods}

The eligibility criterion of this study was the presence of a measurable or evaluable lesion. Other criteria included: a two-week or longer drug withdrawal after previous therapy, adequate bone marrow function, liver function and renal function, 75-year-old or younger age, an expected survival of 3 months or longer, performance status 0-2 and the absence of active double cancer. Informed consent was obtained in writing from the patients enrolled in the study.

Eligible patients who entered the study underwent 2 or more courses of weekly paclitaxel + UFT therapy as the protocol therapy. One course of this regimen took 4 weeks. Paclitaxel was infused intravenously for $60 \mathrm{~min}$ on days 1,8 and 15, and UFT was orally administered daily for 21 days, followed by drug withdrawal for 1 week. As premedication for hypersensitive reactions, dexamethasone $20 \mathrm{mg}$ d.i.v., diphenhydramine $500 \mathrm{mg}$ p.o. and ranitidine $50 \mathrm{mg}$ i.v. were administered $30 \mathrm{~min}$ before paclitaxel administration.

The dose escalation schedule was set as: the initial dose (level 1) was paclitaxel, $80 \mathrm{mg} / \mathrm{m}^{2}$ and UFT, $400 \mathrm{mg} /$ day. At level 2, paclitaxel remained the same, but UFT was increased to $600 \mathrm{mg} /$ day. At level 3, only paclitaxel was increased to $90 \mathrm{mg} / \mathrm{m}^{2}$. When the initial dose was determined to be the maximum tolerated dose (MTD), level 0 and level -1 were set as follows: at level 0 and level -1 , the dose of UFT was fixed at $400 \mathrm{mg} /$ day and paclitaxel was changed to $70 \mathrm{mg} / \mathrm{m}^{2}$ at level 0 and $60 \mathrm{mg} / \mathrm{m}^{2}$ at level -1 (Table I). Dose-limiting toxicity (DLT) was defined in accordance with the National Cancer Institute Common Toxicity Criteria. The criteria were: grade 4 thrombocytopenia, grade 3 pyrexial neutropenia $\left(\geq 38^{\circ} \mathrm{C}\right)$, grade 4 neutropenia that persists for $\geq 4$ days, grade 3-4 peripheral neuropathy and grade 3-4 non-hematological toxicity (excluding depilation, nausea and vomiting). One dose level was assigned to a cohort of 3 patients and when no DLT was noted, the study proceeded to the next dose level. When DLT was noted in $1 / 3,3$ additional patients were assigned to the same level. When DLT was noted in $\geq 2$ patients at the same dose level, the dose was determined to be MTD. A one-level
Table I. Dose escalation scheme.

\begin{tabular}{lcc}
\hline Dose level & Paclitaxel $\left(\mathrm{mg} / \mathrm{m}^{2}\right)$ & UFT $(\mathrm{mg} /$ body $)$ \\
\hline-1 & 60 & 400 \\
0 & 70 & 400 \\
1 & 80 & 400 \\
2 & 80 & 600 \\
3 & 90 & 600 \\
\hline
\end{tabular}

Table II. Patient characteristics.

\begin{tabular}{|c|c|c|}
\hline Characteristics & No. of patients & $\%$ \\
\hline No. of patients entered & 12 & \\
\hline \multicolumn{3}{|l|}{ Age, year } \\
\hline Median & 53 & \\
\hline Range & $40-73$ & \\
\hline \multicolumn{3}{|l|}{ Performance status } \\
\hline 0 & 10 & 83.3 \\
\hline 1 & 2 & 16.7 \\
\hline 2 & 0 & 0.0 \\
\hline \multicolumn{3}{|l|}{ Menopausal status } \\
\hline Pre- & 3 & 25.0 \\
\hline Post- & 9 & 75.0 \\
\hline \multicolumn{3}{|l|}{ No. of metastatic sites involved } \\
\hline 1 & 8 & 66.7 \\
\hline 2 & 4 & 33.3 \\
\hline \multicolumn{3}{|l|}{ Hormone receptor status } \\
\hline \multicolumn{3}{|l|}{ Estrogen or progesterone } \\
\hline Positive receptor & 7 & 58.3 \\
\hline Negative receptor & 5 & 41.7 \\
\hline Unknown & 0 & 0.0 \\
\hline \multicolumn{3}{|l|}{ Prior chemotherapy } \\
\hline Prior adjuvant chemotherapy & 9 & 75.0 \\
\hline $\begin{array}{l}\text { Prior chemotherapy for } \\
\text { metastatic disease }\end{array}$ & 8 & 66.7 \\
\hline Prior anthracycline & 10 & 83.3 \\
\hline Prior taxane & 4 & 33.3 \\
\hline
\end{tabular}

lower dose than MTD was selected as the recommended dose (RD) for the phase II study.

Table III. Toxicity according to dosing level.

\begin{tabular}{ccccc}
\hline Level & PTX/UFT & No. of patients & DLT & No. of patients with DLT \\
\hline-1 & $60 / 400$ & 5 & - & 0 \\
0 & $70 / 400$ & 4 & - & 0 \\
1 & $80 / 400$ & 3 & Liver dysfunction, neutropenia & 2 \\
\hline
\end{tabular}

PTX, paclitaxel; DLT, dose-limiting toxicity; UFT, uracil and tegafur. 
Table IV. Toxicity profiles.

\begin{tabular}{|c|c|c|c|c|c|c|c|c|c|c|}
\hline \multirow[b]{3}{*}{ Toxicity } & \multicolumn{10}{|c|}{ Grade } \\
\hline & \multicolumn{2}{|c|}{0} & \multicolumn{2}{|c|}{1} & \multicolumn{2}{|c|}{2} & \multicolumn{2}{|c|}{3} & \multicolumn{2}{|c|}{4} \\
\hline & No. & $\%$ & No. & $\%$ & No. & $\%$ & No. & $\%$ & No. & $\%$ \\
\hline Leukocytopenia & 7 & 64 & 1 & 9 & 1 & 9 & 2 & 18 & - & \\
\hline Neutropenia & 7 & 64 & 1 & 9 & 1 & 9 & - & & 2 & 18 \\
\hline Thrombocytopenia & 11 & 100 & - & & - & & - & & - & \\
\hline Fever & 11 & 100 & - & & - & & - & & - & \\
\hline Diarrhea & 11 & 100 & - & & - & & - & & - & \\
\hline Alopecia & - & & 7 & 64 & 4 & 36 & - & & - & \\
\hline Neurosensory & 6 & 55 & 5 & 45 & - & & - & & - & \\
\hline Skin & 11 & 100 & - & & - & & - & & - & \\
\hline Stomatitis & 10 & 91 & 1 & 9 & - & & - & & - & \\
\hline Arthralgia & 10 & 91 & 1 & 9 & - & & - & & - & \\
\hline Myalgia & 11 & 100 & - & & - & & - & & - & \\
\hline Liver dysfunction & 10 & 91 & - & & - & & - & & 1 & 9 \\
\hline Hypersensitivity reaction & 10 & 91 & - & & 1 & 9 & - & & - & \\
\hline Fatigue & 9 & 82 & 2 & 18 & - & & - & & - & \\
\hline Appetite loss & 9 & 82 & 2 & 18 & - & & - & & - & \\
\hline Nausea & 9 & 82 & 2 & 18 & - & & - & & - & \\
\hline Headache & 9 & 82 & 2 & 18 & - & & - & & - & \\
\hline Flushing & 8 & 73 & 3 & 27 & - & & - & & - & \\
\hline
\end{tabular}

\section{Results}

Twelve patients were enrolled in this study between September 2000 and September 2002. The median age of the patients was 52.8 years of age (42-67 years) and the performance status (ECOG) was 0 in the 12 patients (Table II). Eleven patients had metastatic breast cancer and 1 patient had local progressive breast cancer. Patients with metastatic breast cancer had previously undergone chemotherapy and 6 of them were on chemotherapy including anthracycline.

Three patients were assigned to level 1. Grade 3 liver dysfunction (increased apartate aminotransferase and alanine aminotransferase) was noted in 1 patient and grade 4 neutropenia was noted in 1 patient, showing that DLT was detected in $2 / 3$ patients. In accordance with the protocol, UFT was fixed at $400 \mathrm{mg} /$ day and paclitaxel was decreased to $60 \mathrm{mg} / \mathrm{m}^{2}$ at level -1 and then increased to $70 \mathrm{mg} / \mathrm{m}^{2}$ at level 0 .

Five patients were assigned to level -1 , and 2 of the patients were handled as dropouts. One dropout developed grade 3 neutropenia in the first course and postponed administration of the drugs. Recovery, however, was delayed and the protocol therapy was discontinued. The safety evaluation committee advised the addition of 1 patient to confirm safety and 1 patient was thus added. However, the fourth patient was also judged as a dropout, since hypersensitive reaction developed immediately after initial administration of paclitaxel. Thus, 5 patients were enrolled. No DLT was noted in 3 patients judged evaluable and the study proceeded to the next step. At level 0 no DLT was noted in any patient. One patient was judged to be a dropout due to grade 4 neutropenia. However, persistence of neutro-
Table V. Overall tumor response.

\begin{tabular}{lccc}
\hline Tumor response & \multicolumn{3}{c}{ Dose level } \\
\cline { 2 - 4 } & $-1(\mathrm{n}=3)$ & $0(\mathrm{n}=3)$ & $1(\mathrm{n}=3)$ \\
\hline $\mathrm{CR}$ & 0 & 0 & 0 \\
PR & 0 & 2 & 1 \\
SD & 3 & 1 & 2 \\
PD & 0 & 0 & 0 \\
CR+PR & 0 & $2(66.7 \%)$ & $1(33.3 \%)$
\end{tabular}

$\mathrm{CR}$, complete response; $\mathrm{PR}$, partial response; $\mathrm{SD}$, stable disease; $\mathrm{PD}$, progressive disease.

penia for 4 days or longer could not be confirmed (Table III). Based on these findings, MTD in this protocol was paclitaxel, $80 \mathrm{mg} / \mathrm{m}^{2}$ and UFT, $400 \mathrm{mg} /$ day; RD was paclitaxel, $70 \mathrm{mg} / \mathrm{m}^{2}$ and UFT, $400 \mathrm{mg} / \mathrm{day}$.

Table IV shows the frequency of the main adverse events. DLT was neutropenia, but was complicated in 1 patient at level 1 and liver dysfunction occurred in 1 patient at level 1 . The incidence of grade 3-4 neutropenia was $18 \%$.

Non-hematological drug-related toxicities were rarely severe and remained easily manageable except liver dysfunction, noted in 1 patient at level 1. Toxicities included alopecia (overall incidence 100\%), neuropathy neurosensory (45\%), stomatitis (9\%), arthralgia (9\%), fatigue (18\%), appetite loss $(18 \%)$, nausea (18\%), headache (18\%) and flushing (27\%). 
The overall effective rate after completion of 2 courses was 33\% (3/9) including three cases of partial responses. The remaining patients presented with stable diseases (SD) and no patient had progressive disease. At dose levels, the effective rate at level 0 , which was RD, was $66.7 \%(2 / 3)$ and continuity was also high (Table $\mathrm{V}$ ). In continuous administration, partial responses were confirmed in some patients after 7 courses and complete response was confirmed in the remaining patients after 4 courses. Regarding the regional effects, the effect was noted in the liver, cervical lymph nodes and local skin.

\section{Discussion}

Our starting hypothesis is that administrating paclitaxel on a weekly basis not only improves the tolerability but in combination with oral UFT may also improve the anticancer effect. The results of our phase I trial, show both of these aspects. At the phase II recommended dose, the regimen was well tolerated and was associated with promising anticancer activity (14).

As chemotherapy for progressive/recurrent breast cancer, the current first choice is combination chemotherapy using multiple drugs, including anthracycline anticancer drugs. After taxan anticancer drugs were introduced, the efficacy of taxans for patients who became resistant to anthracyclines has been reported. Comparative studies, as well as studies on the combination of these anticancer drugs are underway.

Furthermore, weekly administration of paclitaxel in comparison with the standard every-three-week administration was recently investigated. Seidman et al performed a phase II clinical study of the weekly administration of paclitaxel in anthracycline-resistant breast cancer patients and obtained a high effective rate of $53 \%$ (10). Weekly administration was also reported in Japan, where adverse events (peripheral neuropathy and inhibition of the bone marrow) were milder and the effect was higher than those with every-three-week administration (15). According to Kimura et al, when $80 \mathrm{mg} /$ $\mathrm{m}^{2}$ paclitaxel was administered for 3 weeks followed by oneweek withdrawal, a high effective rate of $71.4 \%$ was obtained (16).

5-Fluorouracil is included in combination regimens with cyclophosphamide, doxorubicin and 5-fluorouracil (CAF), as well as cyclophosphamide, epirubicin and 5-fluorouracil $(\mathrm{CEF})$, and is administered intravenously. In contrast, oral 5-fluorouracil anticancer drugs are frequently administered in Japan. UFT is an oral anticancer drug consisting of tegafur and uracil. UFT inhibits the rate-limiting decomposition enzyme DPD, and is called dihydropyrimidine dehydrogenase inhibitory fluoropyrimidine (DIF). The effective rate of UFT was found to be $32 \%$ (16/50) in a Japanese phase II study (10).

Basic investigations of the combination of paclitaxel and UFT using a lung-metastasized breast cancer model have been reported. The concomitant administration of the two drugs inhibited cancer growth without increasing toxicity. Thus, the duration of growth inhibition by paclitaxel alone was short (7-14 days) and re-growth occurred during the administration period, while the combination with UFT inhibited cancer growth for an extensive period of time. Repeated administration of paclitaxel has been reported to induce MDR and resistance, but 5-fluorouracil does not cross-react with MDR.
Thus, the combination of paclitaxel and 5-fluorouracil is a useful one. In addition, DPD activity is higher in metastatic than in primary lesions, suggesting that a DIF UFT is an appropriate 5-fluorouracil anticancer drug in combination with paclitaxel.

In this study, weekly paclitaxel with concomitant UFT was administered. The recommended doses of paclitaxel and UFT were determined to be $70 \mathrm{mg} / \mathrm{m}^{2}$ and $400 \mathrm{mg} /$ day, respectively. As the toxicity profile shows, the major toxicity of this regimen was neutropenia and liver dysfunction, and DLT was neutropenia. Although hypersensitive reaction was noted after the initial administration of paclitaxel in 1 patient, no peripheral nerve toxicity attributable to paclitaxel occurred. This was a phase I study that aimed to determine the recommended dose. However, the number of patients enrolled was small and the effective rate at RD was $66.7 \%$ (2/3), suggesting the usefulness of this regimen.

Based on the results of this study, a phase II study is being performed at the recommended dose determined in the phase I study in patients previously treated with anthracycline. Results of this phase II study are anticipated.

\section{References}

1. A'Hern RP, Smith IE and Ebbs SR: Chemotherapy and survival in advanced breast cancer: the inclusion of doxorubicin in Cooper type regimens. Br J Cancer 67: 801-805, 1993.

2. Fossati R, Confalonieri C, Torri V, Ghislandi E, Penna A, Pistotti V, Tinazzi A and Liberati A: Cytotoxic and hormonal treatment for metastatic breast cancer: a systematic review of published randomized trials involving 31,510 women. J Clin Oncol 16: 3439-3460, 1998.

3. Paterson AH, Szafran O, Cornish F, Lees AW and Hanson J: Effect of chemotherapy on survival in metastatic breast cancer. Breast Cancer Res Treat 1: 357-363, 1981.

4. Pentheroudakis G, Razis E, Athanassiadis A, Pavlidis N and Fountzilas G: Paclitaxel-carboplatin combination chemotherapy in advanced breast cancer: accumulating evidence for synergy, efficacy, and safety. Med Oncol 23: 147-160, 2006.

5. Polyzos A, Tsavaris N, Kosmas C, Gogas H, Toufexi H, Kosmidis C, Markopoulos C, Giannopoulos A, Papadopoulos O, Stamatiadis D and Kouraklis G: Full dose paclitaxel plus vinorelbine as salvage chemotherapy in anthracycline-resistant advanced breast cancer: a phase II study. J Chemother 15: 607-612, 2003.

6. Seo JH, Oh SC, Choi CW, Kim BS, Shin SW, Kim YH, Kim JS, Kim AR, Lee JB and Koo BH: Phase II study of a gemcitabine and cisplatin combination regimen in taxane resistant metastatic breast cancer. Cancer Chemother Pharmacol 59: 269-274, 2007.

7. Holmes FA, Walters RS, Theriault RL, Forman AD, Newton LK, Raber MN, Buzdar AU, Frye DK and Hortobagyi GN: Phase II trial of taxol, an active drug in the treatment of metastatic breast cancer. J Natl Cancer Inst 83: 1797-1805, 1991.

8. Seidman AD, Reichman BS, Crown JP, Yao TJ, Currie V, Hakes TB, Hudis CA, Gilewski TA, Baselga J, Forsythe P, et al: Paclitaxel as second and subsequent therapy for metastatic breast cancer: activity independent of prior anthracycline response. J Clin Oncol 13: 1152-1159, 1995.

9. Ito Y, Horikoshi N, Watanabe T, Sasaki Y, Tominaga T, Okawa T, Tabei T, Kuraishi Y, Tamura K, Abe R, Kitajima M, Yamaguchi S, Kobayashi T, Koyama H, Orita K, Takashima S, Nomura Y and Ogawa M: Phase II study of paclitaxel (BMS-181339) intravenously infused over 3 hours for advanced or metastatic breast cancer in Japan. BMS-181339 Breast Cancer Study Group. Invest New Drugs 16: 183-190, 1998.

10. Seidman AD, Hudis CA, Albanell J, Tong W, Tepler I, Currie V, Moynahan ME, Theodoulou M, Gollub M, Baselga J and Norton L: Dose-dense therapy with weekly 1-hour paclitaxel infusions in the treatment of metastatic breast cancer. J Clin Oncol 16: 3353-3361, 1998. 
11. Lokich JJ, Ahlgren JD, Gullo JJ, Philips JA and Fryer JG: A prospective randomized comparison of continuous infusion fluorouracil with a conventional bolus schedule in metastatic colorectal carcinoma: a Mid-Atlantic Oncology Program Study. J Clin Oncol 7: 425-432, 1989.

12. Ota $\mathrm{K}$, Taguchi $\mathrm{T}$ and Kimura $\mathrm{K}$ : Report on nationwide pooled data and cohort investigation in UFT phase II study. Cancer Chemother Pharmacol 22: 333-338, 1988.

13. Tashiro H, Nomura Y and Ohsaki A: A double blind comparative study of tegafur (FT) and UFT (a combination of tegafur and uracil) in advanced breast cancer. Jpn J Clin Oncol 24: 212-217, 1994.
14. Passardi A, Maltoni R, Milandri C, Cecconetto L, Massa I, Zoli W, Tesei A, Fabbri F, Nanni O and Amadori D: Phase I study of paclitaxel and uracil plus tegafur combination in patients with pretreated metastatic breast cancer: drug sequencing based on preclinical modelling studies. Oncology 72: 118-124, 2007.

15. Kim R, Osaki A and Toge T: Feasibility and therapeutic efficacy of weekly 1-h low-dose paclitaxel infusion for relapsed breast cancer. Oncol Rep 10: 145-150, 2003.

16. Kimura M, Koida T and Yanagita Y: Weekly administration of paclitaxel for advanced or metastatic breast cancer-short-course premedications for outpatients. Gan To Kagaku Ryoho 27: 1703-1708, 2000. 